\title{
Constitutive model for shear-thickening suspensions: Predictions for steady shear with superposed transverse oscillations
}

\author{
J. J. J. Gillissen, C. Ness, J. D. Peterson, H. J. Wilson, and M. E. Cates
}

Citation: Journal of Rheology 64, 353 (2020); doi: 10.1122/1.5129657

View online: https://doi.org/10.1122/1.5129657

View Table of Contents: https://sor.scitation.org/toc/jor/64/2

Published by the The Society of Rheology

\section{ARTICLES YOU MAY BE INTERESTED IN}

Preface: Physics of dense suspensions

Journal of Rheology 64, 223 (2020); https://doi.org/10.1122/8.0000016

Investigating the nature of discontinuous shear thickening: Beyond a mean-field description

Journal of Rheology 64, 329 (2020); https://doi.org/10.1122/1.5132317

A hydrodynamic model for discontinuous shear-thickening in dense suspensions

Journal of Rheology 64, 379 (2020); https://doi.org/10.1122/1.5134036

Experimental test of a frictional contact model for shear thickening in concentrated colloidal suspensions Journal of Rheology 64, 267 (2020); https://doi.org/10.1122/1.5129798

Shear thickening in dense non-Brownian suspensions: Viscous to inertial transition

Journal of Rheology 64, 227 (2020); https://doi.org/10.1122/1.5129680

Fluctuations at the onset of discontinuous shear thickening in a suspension

Journal of Rheology 64, 309 (2020); https://doi.org/10.1122/1.5131740
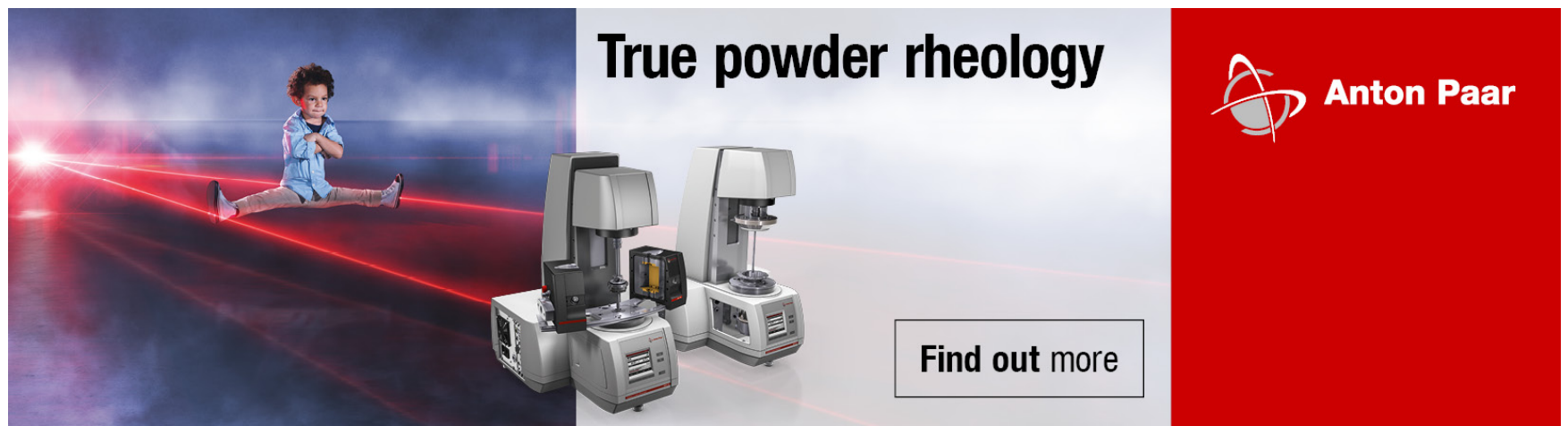


\title{
Constitutive model for shear-thickening suspensions: Predictions for steady shear with superposed transverse oscillations
}

\author{
J. J. J. Gillissen, ${ }^{1, a)}$ C. Ness, ${ }^{2}$ J. D. Peterson, ${ }^{3}$ H. J. Wilson, ${ }^{1}$ and M. E. Cates ${ }^{3}$ \\ ${ }^{1}$ Department of Mathematics, University College London, Gower Street, London WC1E 6BT, United Kingdom \\ ${ }^{2}$ Department of Chemical Engineering and Biotechnology, University of Cambridge, Cambridge CB3 OAS, \\ United Kingdom \\ ${ }^{3}$ DAMTP, Centre for Mathematical Sciences, University of Cambridge, Cambridge CB3 OWA, United Kingdom
}

(Received 30 September 2019; final revision received 10 January 2020; published 4 March 2020)

\begin{abstract}
We recently developed a tensorial constitutive model for dense, shear-thickening particle suspensions that combines rate-independent microstructural evolution with a stress-dependent jamming threshold. This gives a good qualitative account for reversing flows, although it quantitatively overestimates structural anisotropy [J. J. J. Gillissen et al., Phys. Rev. Lett. 123(21), 214504 (2019)]. Here, we use the model to predict the unjamming effect of superposed transverse oscillations on a steady shear flow in the thickened regime [N. Y. C. Lin et al., Proc. Natl. Acad. Sci. U.S.A. 113, 10774 (2016)]. The model successfully reproduces the oscillation-mediated viscosity drop observed experimentally. We compare the time-dependent components of the stress and microstructure tensors to discrete-element simulations. Although the model correctly captures the main qualitative behavior, it generally over-predicts the microstructural anisotropy in steady shear, and it under-predicts the number of particle contacts in oscillating shear. It also does not fully capture the correct variation in phase angle between the transverse component of the microstructure and the shear rate oscillations as the amplitude of the latter is increased. These discrepancies suggest avenues for future improvements to the model. (C) 2020 The Society of Rheology. https://doi.org/10.1122/1.5129657
\end{abstract}

\section{INTRODUCTION}

Dense suspensions of hard particles in a viscous solvent are found in many application domains including the construction industry, food production, and pharmaceuticals. Such materials, which have a solid volume fraction $\phi \gtrsim 0.4$, often exhibit shear thickening, an increase (continuous or discontinuous) in viscosity under increasing shear rate $\dot{\gamma}$. Understanding and controlling this distinctive rheological behavior is key to operating efficient and reliable processes and has been the subject of many studies during the past three decades.

Recent numerical [1,2] and experimental [3-5] data provide evidence that, in contrast to scenarios envisaged in much of the prior literature [6], shear thickening in non-Brownian, noninertial suspensions is caused by the onset of direct interparticle contacts that are frictional in character. In addition to tangential contact friction forces, shear thickening may also arise due to tangential lubrication forces that act between asperities on the opposing particle surfaces [7]. In both (the contact friction and the lubrication) scenarios, shear thickening results from constraints due to tangential forces. Although our theoretical treatment of both scenarios would be similar, we follow the contact friction narrative in this work. Experimental data [3] for the steady-state viscosity as a function of shear rate are well described by the theory of Wyart and Cates (WC) [8], in which

Note: This paper is part of the special issue on Physics of Dense Suspensions. ${ }^{a)}$ Author to whom correspondence should be addressed: electronic mail: jurriaangillissen@gmail.com the appearance of such contacts under steady flow is governed by a competition between a short-ranged interparticle repulsion, of maximum force $F^{*}$, and the macroscopic particle pressure $\Pi=-\operatorname{Tr} \boldsymbol{\Sigma} / 3$, with $\boldsymbol{\Sigma}$ being the particle stress tensor. In suspensions of strictly hard spheres, whether frictional or not, dimensional analysis predicts rate-independent rheology, i.e., $\Sigma \propto \dot{\gamma}$ [9]. However, the presence of a characteristic force scale $F^{*}$ allows the physics to depend on a dimensionless shear rate,

$$
\dot{\gamma}_{r}=\frac{\dot{\gamma} \eta_{s}}{\Pi^{*}},
$$

with $\eta_{s}$ being the solvent viscosity, $a$ the particle radius, and $\Pi^{*} \sim F^{*} / a^{2}$ the so-called "onset stress." At small flow rates, where $\Pi<\Pi^{*}$, the typical interparticle force remains less than $F^{*}$, and particles remain separated by lubrication films [10]. At large flow rates, where $\Pi>\Pi^{*}$, lubrication films break down and particles enter into solid-solid frictional contact. Friction restricts particle sliding so that steady flow requires more tortuous particle trajectories, leading to an increase in the suspension viscosity.

Based upon this principle, WC write, for a steady $x y$ shear flow, a relation between the nondimensionalized suspension viscosity $\eta_{r}=\Sigma_{x y} /\left(\eta_{s} \dot{\gamma}\right)$, the volume fraction $\phi$, and the dimensionless shear rate $\dot{\gamma}_{r}$. The relation is based on the Krieger-Dougherty equation [11],

$$
\eta_{r} \sim\left(1-\phi / \phi^{J}\right)^{-2}
$$

where $\phi^{J}$ is the volume fraction at jamming. WC introduce rate-dependence by relating $\phi^{J}$ to the onset of friction 
described above, noting that friction imposes additional constraints at particle contact, reducing the number of contacts per particle (or coordination number, $Z$ ) required for jamming. Moreover, they effectively assume that the steadystate microstructure itself is friction-independent so that the stress dependence enters not by changes in $Z$ itself, but by changes in the jamming point $Z^{J}$, which, for spheres in three dimensions, can vary between four (all contacts rolling) and six (all contacts sliding). This assumption causes the steadystate $Z$ value to depend solely on volume fraction, so reducing $Z^{J}$ is equivalent to reducing $\phi^{J}$. Hence, WC postulated

$$
\phi^{J}(f)=\phi_{1}^{J}(1-f)+\phi_{2}^{J} f, \quad f(\Pi)=\exp \left(-\Pi^{*} / \Pi\right),
$$

where $f(\Pi)$ is the fraction of contacts that are constrained by friction to roll rather than slide. \{The particular form of $f(\Pi)$ is relatively unimportant; the above choice was made later, on empirical grounds, in [12].\} The limiting volume fractions at which frictionless and fully frictional packings become rigid, $\phi_{1}^{J} \approx 0.64$ and $\phi_{2}^{J} \approx 0.57$, respectively (in $3 \mathrm{D}$ ), are generally agreed upon in the literature.

Although Eqs. (2) and (3) have a featureless, monotonic dependence of $f$ on $\Pi$, they predict flow curves (shear stress versus shear rate) that, depending on $\phi$, imply continuous and discontinuous shear thickening as well as "full jamming" (whereby the viscosity is infinite above a $\Pi$ threshold comparable in magnitude to $\Pi^{*}$ ). In particular, discontinuous shear thickening arises as a jump between the lower and upper branches of a flow curve that is everywhere smooth, but $S$-shaped [8].

The WC theory agrees well with experiments and particlebased simulations under steady and homogeneous conditions [3], at least for modest particle size polydispersity [13]. Its predictions of nonmonotonic flow curves also signal the presence of steady shear-banding and other instabilities leading to spatiotemporal variations of the flow state [12]. However, it neither makes predictions for unsteady flow nor does it quantitatively address the tensorial character of the stress tensor. In other words, WC did not offer a full constitutive model for shear-thickening suspensions. At first sight, one might consider applying the WC equations (2) and (3) at each point in time during an evolving flow, but the resulting implicit assumption that the coordination number $Z$ depends only upon $\phi$ is clearly invalidated by the flow-history dependence of the microstructure.

To address this, we have recently formulated a tensorial constitutive model in which the viscosity depends on a timeevolving "jamming coordinate" $\xi$, defined in Eq. (37) below, which can take over the role played by $\phi$ in the WC theory [14]. Although $\xi$ is effectively a proxy for a time-evolving microscopic coordination number $Z$, the jamming coordinate is computable from the coarse-grained microstructure $\langle\boldsymbol{n n}\rangle$ [see Eq. (24) below], allowing closure of our equations at that level. Our model includes a microstructure-tensor evolution equation, which was derived previously for rate-independent suspensions [15] from which $\xi$ is computed, with key intuitions for shear-thickening suspensions as described in the scalar and time-independent WC approach [8]. These are the (linear) interpolation between jamming conditions as a function of $f(\Pi)$ and the singular (Krieger-Dougherty) dependence of viscosity on $1-\xi / \xi^{J}$, where $\xi^{J}$ is the jamming coordinate at the jamming point which is defined in Eq. (9) below. In [14], we demonstrated that the new constitutive model performs well under shear reversal, correctly predicting the discontinuous drop in $\eta_{r}=\Sigma_{x y} /\left(\dot{\gamma} \eta_{s}\right)$ at very small strain and its subsequent smooth recovery. Abrupt flow reversals of this kind represent important test cases, which in the literature have been used to gain insight into historydependent microstructure [16] and to distinguish the contact and hydrodynamic contributions to suspension stress $[4,17,18]$. Their challenging character for constitutive models has been previously pointed out $[19,20]$.

In the present work, we further test the new constitutive model by addressing the case of a steady shear flow (shear rate $\dot{\gamma}$ ) with superposed transverse shear flow oscillations. The latter is at $90^{\circ}$ to the steady flow and has frequency $\omega$ and strain amplitude $\gamma$ [Fig. 1(a)]. The steady shear has flow in $x$ and gradient in $y$, while the transverse oscillations are in $\pm z$ with gradient in $y$. As opposed to an abrupt flow reversal, such flows constitute a continuous family of time-dependent, controlled distortions to the steady shear flow, characterized by their amplitude $\gamma$ \{which, as discussed later, we fix at $\gamma=0.01$ following the experiments of [21] $\}$ and a dimensionless frequency $\dot{\gamma}_{\perp}$, see Fig. 1(b),

$$
\dot{\gamma}_{\perp}=\frac{\omega \gamma}{\dot{\gamma}}
$$

Recent experiments [21] and discrete-element method (DEM) simulations [22] demonstrate that the oscillations break up the fragile jammed network of interparticle contacts [23]. This breakup can substantially reduce the viscosity in systems with $\phi$ just below $\phi_{2}^{J}$ (where discontinuous shear thickening arises). Moreover, for systems that are fully jammed $\left(\phi>\phi_{2}^{J}, \Pi \gg \Pi^{*}\right)$, the viscosity falls from the (effectively infinite) jammed value to a finite one. The mechanism behind the loss of contacts has been explained from the perspectives of force chain dynamics [21] and random organization [22]. Transverse shear flow oscillations may enhance suspension flow in practical applications [24]. In addition, this flow configuration also offers a subtle and
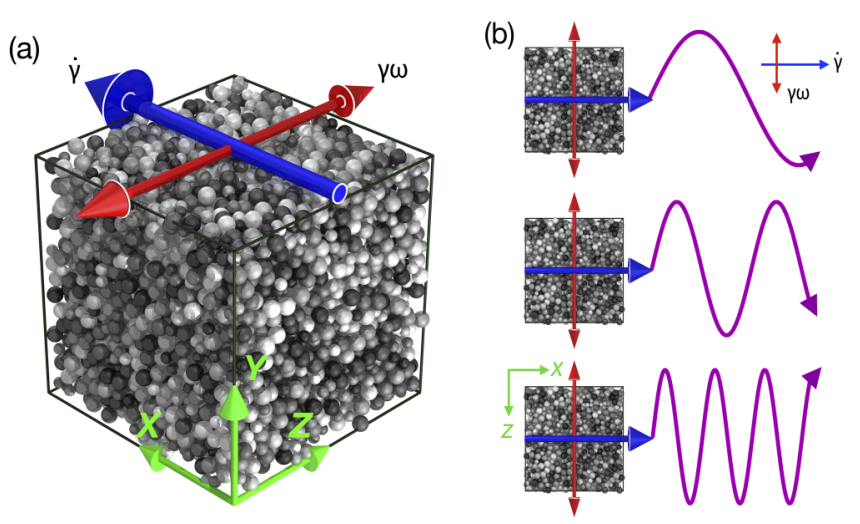

FIG. 1. (a) Model suspension with a coordinate definition in green. Blue arrow indicates steady shear flow; red arrows indicate superposed transverse oscillatory shear flow. (b) Top view of model suspension showing examples of shearing trajectories with different $\dot{\gamma}_{\perp}=\gamma \omega / \dot{\gamma}$. 
challenging test case for constitutive models for suspension microstructure and stress.

The remainder of the paper is organized as follows. Section II presents a self-contained derivation of our constitutive model, including those parts first presented in [25] and [15] as well as the new features added in [14] to address shear thickening. In Sec. III, we give brief details of the discrete-element simulation model from which we generate test data in the chosen flow geometry. In Sec. IV, we compare the results of the constitutive model to those of the DEM across a range of $\dot{\gamma}_{r}$ and $\dot{\gamma}_{\perp}$. Our conclusions are given in Sec. V.

\section{CONSTITUTIVE MODEL}

We consider a collection of non-Brownian spheres of radius $a$, suspended at a volume fraction of $\phi$ and a number density of $n=\phi /\left(\frac{4}{3} \pi a^{3}\right)$ in a fluid of density $\rho$ and viscosity $\eta_{s}$. The volume-averaged fluid velocity is $\boldsymbol{U}$, and the fluid velocity gradient and deformation tensors are given by $\boldsymbol{L}=\nabla \boldsymbol{U}^{T}$ and $\boldsymbol{E}=\frac{1}{2}\left(\boldsymbol{L}+\boldsymbol{L}^{T}\right)$, respectively. The particle Reynolds number is $\dot{\gamma} \rho a^{2} / \eta_{s} \ll 1$ (allowing inertia to be neglected), and $\phi$ is assumed sufficiently large that hydrodynamic interactions between particles can effectively be modeled as lubrication forces.

Below, we derive an equation for the particle stress tensor $\boldsymbol{\Sigma}$, which is based on an equation of motion for the statistics of the particle pair separation unit vector $\boldsymbol{n}$, which is encoded in the second moment $\langle\boldsymbol{n} \boldsymbol{n}\rangle$ of the distribution function $\Psi(\boldsymbol{n})$. In Sec. II A, we derive an equation of motion for $\boldsymbol{n}$ for a single particle pair. In Sec. II B, we use this equation to derive the equation of motion for $\langle\boldsymbol{n} \boldsymbol{n}\rangle$, and in Sec. II C, we relate $\boldsymbol{\Sigma}$ to $\langle\boldsymbol{n n}\rangle$.

\section{A. Particle pair motion}

Following [15], we start by writing an equation of motion for the connection vector $\boldsymbol{r}$ of a particle pair, that points to a so-called "test particle" (TP) from a so-called "pairing particle" (PP). Under the conditions given above, Newton's equation of motion, applied to the TP, reads

$$
\begin{aligned}
\boldsymbol{0}= & C_{1} a \eta_{s}(\boldsymbol{L} \cdot \boldsymbol{r}-\dot{\boldsymbol{r}}) \\
& -C_{2} a \eta_{s}(\dot{\boldsymbol{r}} \cdot \boldsymbol{n}) \boldsymbol{n}+C_{3} a^{2} \eta_{s} \dot{\gamma} \Theta(2 a-r) \boldsymbol{n} .
\end{aligned}
$$

Here, $\boldsymbol{n}=\boldsymbol{r} / r$ is the interaction unit vector, $r=|\boldsymbol{r}|, C_{1,2,3}$ are dimensionless prefactors, specified below, and $\Theta(u)$ is the Heaviside step-function, with $\Theta(u<0)=0, \Theta(u \geq 0)=1$. For strictly hard-core particles, the $\Theta$ function counts contacts; in systems where hard-core contact is replaced by particle overlaps (as is often done in simulations), it continues to do so. Note that in [15], we wrote $C_{1} / 2$ instead of $C_{1}$.

The $C_{1}$-term in Eq. (5) is the interaction force between the TP and the background mixture, which is proportional to the difference between the TP velocity $\dot{\boldsymbol{r}}$ and the mixture velocity at the TP location, $\boldsymbol{L} \cdot \boldsymbol{r}$. The $C_{2}$-term in Eq. (5) is the lubrication interaction force between the TP and the PP. The leading order contribution to the lubrication force is $\sim a^{2}(r-2 a)^{-1} \eta_{s}(\dot{\boldsymbol{r}} \cdot \boldsymbol{n}) \boldsymbol{n}$, where $(r-2 a)$ is the interparticle gap [26]. In order to arrive at tractable expressions for the suspension microstructure and stress [Eqs. (24) and (35)], we have replaced the factor $a(r-2 a)^{-1}$ with its averaged value $C_{2}$, which is taken to obey the Krieger-Dougherty form [11]

$$
C_{2} \sim\left(1-\phi / \phi_{1}^{J}\right)^{-2}
$$

where $\phi_{1}^{J}$ is the particle volume fraction at random close packing.

The $C_{3}$-term in Eq. (5) is the contact force between the TP and the PP. The expression for the contact force assumes that this force (i) aligns with $\boldsymbol{n}$, (ii) acts on the particle surface, (iii) scales as a viscous force $\sim a^{2} \eta_{s} \dot{\gamma}$, and (iv) is proportional to a dimensionless prefactor $C_{3}$.

Note that in treating $C_{1,2,3}$ as constants, independent of a local microstructure, we have already used a mean-field type of averaging. (This applies particularly for the constraint force $C_{3}$ which, at a particular contact, can take any positive value to balance the other forces acting.) After such averaging, the interaction force between the TP and the background must balance the dominant term of the interaction force with the PP so that, in magnitude,

$$
C_{1} \sim \max \left(C_{2}, C_{3}\right) .
$$

Note that there are no tangential (lubrication or contact friction) forces in Eq. (5) and we do not consider the torque balance. When considering particle motion, the omission of tangential contact forces, caused by friction, is justified by the assumption, inherited from the WC theory, that microstructural evolution is not itself altered by frictional forces (although the stress for a given microstructure and flow is strongly altered). This assumption is further justified by observations from DEM (i) that the magnitude of the tangential contact forces is small compared to that of the normal contact forces, even under shear-thickened conditions [27] and (ii) that the microstructure is nearly unaffected by shear thickening [14]. Indirectly, the tangential contact friction forces are important as they affect the suspension rheology by imposing constraints on the particle motion [8]. The resulting increase in the suspension viscosity is, however, mainly supported by the normal contact forces. Therefore, although we exclude the tangential contact friction forces in Eq. (5), we indirectly account for these forces by incorporating the following jamming behavior in the prefactor $C_{3}$ for the normal contact forces:

$$
C_{3} \sim\left(1-\xi / \xi^{J}\right)^{-2},
$$

which depends on the jamming coordinate $\xi$, a mesoscopic quantity, defined in Eq. (37) below. The jamming coordinate $\xi$ serves as a proxy for the coordination number $Z$, as $\phi$ does in the steady-state WC theory. Although the numerical values of $\xi$ and $Z$ differ, as exemplified in Eq. (39) below, $\xi$ plays a similar role as $Z$, by defining a distance to the jamming point, i.e., $C_{3}$ diverges when $\xi$ reaches its jamming limit $\xi^{J}$ [Eq. (8)]. This jamming limit $\xi^{J}$ is, in turn, assumed to decrease from a larger value $\xi_{1}^{J}$ to a smaller value $\xi_{2}^{J}$, when the system transitions from "lubricated" to "frictional." This transition is encoded in the fraction $f$ of frictional contacts, which smoothly increases from zero to one as the particle pressure in the system, П, passes through the onset 
threshold $\Pi^{*} \sim F^{*} / a^{2}$. Here, $F^{*}$ is the maximum force sustainable by the short-range repulsive interactions,

$$
\xi^{J}=\xi_{1}^{J}(1-f)+\xi_{2}^{J} f, \quad f=\exp \left(-\Pi^{*} / \Pi\right) .
$$

For simplicity, we have adopted the same functional form for $f(\Pi)$ as in Eq. (3).

The normal and tangential components of the interparticle velocity $\dot{\boldsymbol{r}}$ are readily obtained by projecting Eq. (5) onto the relevant directions,

$$
\dot{\boldsymbol{r}} \cdot \boldsymbol{n}=\frac{C_{1}}{C_{1}+C_{2}} \boldsymbol{E}: \boldsymbol{r n}+\frac{C_{3}}{C_{1}+C_{2}} \dot{\gamma} a \Theta(2 a-r),
$$

and, with $\boldsymbol{\delta}$ being the unit tensor,

$$
\dot{\boldsymbol{r}} \cdot(\boldsymbol{\delta}-\boldsymbol{n n})=\boldsymbol{L} \cdot \boldsymbol{r} \cdot(\boldsymbol{\delta}-\boldsymbol{n n}) .
$$

\section{B. Microstructure evolution}

Again following [15], we now introduce the distribution function $\Psi(\boldsymbol{r})$ of the particle-pair separation vector $\boldsymbol{r}$, which evolves according to the Smoluchowski equation for the twoparticle configuration space,

$$
\partial_{t} \Psi+\partial_{k}\left(\dot{r}_{k} \Psi\right)=0
$$

where $\partial_{k}=\partial / \partial r_{k}$. Because the typical spacing between the particles $\epsilon$ is small compared to the particle radius $a$, the anisotropy in $\Psi(\boldsymbol{r})$ is relegated to the so-called coarsegraining shell $2 a<r<2 a+\epsilon$, where $\epsilon$ is related to $\phi$ via $[a /(a+\epsilon)]^{3} \sim \phi / \phi_{1}^{J}$, i.e.,

$$
\frac{\epsilon}{a} \sim 1-\phi / \phi_{1}^{J} .
$$

By assuming that the number of interactions in the coarse-graining shell is $\sim \phi$, we see that $\Psi(\boldsymbol{r}) \sim$ [number of interactions]/ [volume of shell] $\sim \phi /\left(a^{2} \epsilon\right)$. Outside the coarse-graining shell, steric constraints are dominant, and $\Psi\left(r=[2 a+\epsilon]^{+}\right)=\Psi^{\text {outer }}$ is assumed isotropic. By continuity, we write this as

$$
\Psi^{\text {outer }}=\frac{\phi}{4 \pi a^{2} \epsilon} .
$$

Equation (14) suppresses an order-unity prefactor that can, however, be absorbed into other constants appearing below.

Next, we derive the evolution equation for the secondorder orientation moments $\langle\boldsymbol{n n}\rangle$ of the distribution function $\Psi(\boldsymbol{r})$ in the coarse-graining shell, by inserting Eqs. (10) and (11) into Eq. (12), multiplying the result with $\boldsymbol{n n}$, applying the following, so-called coarse-graining operator $\langle\cdots\rangle$,

$$
\langle\cdots\rangle=\int_{r=2 a}^{r=2 a+\epsilon} \Psi(\boldsymbol{r}) \cdots d^{3} \boldsymbol{r},
$$

and approximating $\boldsymbol{r} \approx 2$ an [15],

$$
\begin{aligned}
\partial_{t}\langle\boldsymbol{n n}\rangle= & \boldsymbol{L} \cdot\langle\boldsymbol{n n}\rangle+\langle\boldsymbol{n n}\rangle \cdot \boldsymbol{L}^{T}-2 \boldsymbol{L}:\langle\boldsymbol{n n n n}\rangle \\
& -\frac{2 a C_{1}}{C_{1}+C_{2}} \boldsymbol{E}: \oint_{r=2 a+\epsilon} \Psi(\boldsymbol{r}) \boldsymbol{n n n n n} d^{2} \boldsymbol{r} .
\end{aligned}
$$

The boundary surface integral in Eq. (16) corresponds to an orientation probability flux between the coarse-graining shell $2 a<r<2 a+\epsilon$ and the outer shell $r>2 a+\epsilon$. This flux is carried by the rate of strain tensor $\boldsymbol{E}=\boldsymbol{E}_{c}+\boldsymbol{E}_{e}$, which is decomposed into its positive and negative eigenparts. For instance, in a simple $x y$-shear flow,

$$
\boldsymbol{E}_{e}=\frac{1}{2} \dot{\gamma} \boldsymbol{n}_{e} \boldsymbol{n}_{e}, \quad \boldsymbol{E}_{c}=-\frac{1}{2} \dot{\gamma} \boldsymbol{n}_{c} \boldsymbol{n}_{c},
$$

where $\pm \frac{1}{2} \dot{\gamma}$ are the expansive and the compressive eigenvalues of $\boldsymbol{E}$ and $\boldsymbol{n}_{e}=(1,1,0) / \sqrt{2}$ and $\boldsymbol{n}_{c}=(1,-1,0) / \sqrt{2}$ are the corresponding eigenvectors.

The positive (extensional) eigenpart $\boldsymbol{E}_{e}$ and negative (compressive) eigenpart $\boldsymbol{E}_{c}$ correspond to an outward and an inward probability flux between the coarse-graining shell and the outer shell, respectively. Note that the contact force $\left[C_{3}\right.$-term in Eq. (5)] does not enter Eq. (16); this reflects the fact that, for impenetrable particles, there is no probability flux across the inner surface of the coarse-graining shell at $r=2 a$. Consequently, within our model, the evolution of the coarse-grained microstructure tensor $\langle\boldsymbol{n n}\rangle$ is not directly sensitive to contact forces.

With these assumptions, the surface integral in Eq. (16) can now be recast as [15]

$$
\begin{aligned}
\boldsymbol{E} & : \oint_{r=2 a+\epsilon} \Psi(\boldsymbol{r}) \boldsymbol{n n n n n} d^{2} \boldsymbol{r} \\
& =\boldsymbol{\epsilon}^{-1} \boldsymbol{E}_{e}:\langle\boldsymbol{n n n n n}\rangle+\boldsymbol{\epsilon}^{-1} \boldsymbol{E}_{c}:\langle\boldsymbol{n n n n n}\rangle^{\text {outer }},
\end{aligned}
$$

where $\langle\boldsymbol{n n n n n}\rangle$ and $\langle\boldsymbol{n n n n n}\rangle^{\text {outer }}$ are the orientation moments, evaluated inside the coarse-graining shell and on the outside of the coarse-graining shell [Eq. (14)], respectively. Combining Eqs. (16) and (18), we obtain the following coarse-grained microstructure evolution equation:

$$
\begin{aligned}
\partial_{t}\langle\boldsymbol{n n}\rangle= & \boldsymbol{L} \cdot\langle\boldsymbol{n n}\rangle+\langle\boldsymbol{n n n}\rangle \cdot \boldsymbol{L}^{T}-2 \boldsymbol{L}:\langle\boldsymbol{n n n n n}\rangle \\
& -\beta\left[\boldsymbol{E}_{e}:\langle\boldsymbol{n n n n n}\rangle+\boldsymbol{E}_{c}:\langle\boldsymbol{n n n n n}\rangle^{\text {outer }}\right],
\end{aligned}
$$

where $\beta$ is referred to as the microstructure association rate,

$$
\beta=\frac{2 a C_{1}}{\epsilon\left(C_{1}+C_{2}\right)},
$$

which controls the rate of particle pair association and dissociation. The physical importance of $\beta$ is that its inverse sets a strain scale for structural evolution. On geometrical grounds, $\beta$ should depend on $\phi$ so as to diverge at random close packing $\phi_{1}^{J}$. To determine the dependence of $\beta$ on $\phi$, we make use of Eq. (13). We furthermore see from Eq. (7) that $C_{1} \gtrsim \mathrm{C}_{2}$ such that $C_{1}+C_{2} \sim C_{1}$. Inserting these approximations into Eq. (20), we find

$$
\beta=\frac{\beta_{0}}{1-\phi / \phi_{1}^{J}},
$$

with $\beta_{0}$ being a tuneable parameter. This shows that $\beta$ is roughly constant in the region just below $\phi_{2}^{J}$ where shear thickening is seen. In [14], we determine $\beta$ by matching in 
this region the constitutive model to DEM simulation data after a reversal of steady shear.

The first line of Eq. (19) describes the rotational advection of the contact vectors $\boldsymbol{n}$, whereas the second line corresponds to the association and dissociation of interacting particle pairs by the action of compressive and extensional flow deformations that, respectively, push particles together and pull them apart. More specifically, the compressive rate of strain $\boldsymbol{E}_{c}$ advects, into the coarse-graining shell, the isotropic exterior distribution of noncontacting particles, importing preferentially along the compression axis (or axes). In contrast, the extensional rate of strain $\boldsymbol{E}_{e}$ advects the anisotropically distributed existing contacts out of the coarse-graining shell, exporting preferentially along the extension axis (or axes).

Equation (19) contains the fourth order moment of the inner probability density function $\langle\boldsymbol{n n n n}\rangle$ and of the isotropic outer distribution function $\langle\boldsymbol{n} n \boldsymbol{n n n}\rangle^{\text {outer }}$. We next express $\langle\boldsymbol{n n n n}\rangle$ in terms of $\langle\boldsymbol{n n}\rangle$, using the linear closure model of Hinch and Leal, which is accurate for microstructures that are relatively close to isotropy [28],

$$
\begin{aligned}
\left\langle n_{i} n_{j} n_{k} n_{l}\right\rangle= & -\frac{1}{35}\left\langle n_{m} n_{m}\right\rangle\left(\delta_{i j} \delta_{k l}+\delta_{i k} \delta_{j l}+\delta_{i l} \delta_{j k}\right) \\
& +\frac{1}{7}\left(\delta_{i j}\left\langle n_{k} n_{l}\right\rangle+\delta_{i k}\left\langle n_{j} n_{l}\right\rangle+\delta_{i l}\left\langle n_{j} n_{k}\right\rangle\right. \\
& \left.+\left\langle n_{i} n_{j}\right\rangle \delta_{k l}+\left\langle n_{i} n_{k}\right\rangle \delta_{j l}+\left\langle n_{i} n_{l}\right\rangle \delta_{j k}\right) .
\end{aligned}
$$

The same closure, when applied to the isotropic outer distribution function $\Psi^{\text {outer }}$ [Eq. (14)], reduces to

$$
\begin{aligned}
\langle\boldsymbol{n n n n n}\rangle^{\text {outer }} & =\phi \oint(4 \pi)^{-1} n_{i} n_{j} n_{k} n_{l} d^{2} \boldsymbol{n} \\
& =\frac{\phi}{15}\left(\delta_{i j} \delta_{k l}+\delta_{i k} \delta_{j l}+\delta_{i l} \delta_{j k}\right) .
\end{aligned}
$$

By combining Eqs. (19) and (23), we finally arrive at the closed Gillissen-Wilson equation for microstructural evolution [15],

$$
\begin{aligned}
\partial_{t}\langle\boldsymbol{n n n}\rangle= & \boldsymbol{L} \cdot\langle\boldsymbol{n n n}\rangle+\langle\boldsymbol{n n n}\rangle \cdot \boldsymbol{L}^{T}-2 \boldsymbol{L}:\langle\boldsymbol{n n n n n}\rangle \\
& -\beta\left[\boldsymbol{E}_{e}:\langle\boldsymbol{n n n n n}\rangle+\frac{\phi}{15}\left(2 \boldsymbol{E}_{c}+\operatorname{Tr}\left(\boldsymbol{E}_{c}\right) \boldsymbol{\delta}\right)\right],
\end{aligned}
$$

in which $\langle\boldsymbol{n n n n}\rangle$ is now shorthand for the right hand side (rhs) of Eq. (22).

A significant novelty of Eq. (24) is its separate linearity in the compressive and extensional components of the rate of strain tensor, making it overall nonlinear in the rate of strain and thus distinct from various previous models that failed to adequately predict reversal flows without excessive parameters [20]. On reversal, the compressive and extensional components interchange so that contacts that were being pushed together are now pulled apart.

\section{Particle stress and contacts}

A two-body approximation for the particle stress reads

$$
\boldsymbol{\Sigma}=n\langle\boldsymbol{F r}\rangle,
$$

where $\langle\cdots\rangle$ is the coarse-graining operator defined in Eq. (15) and $\boldsymbol{F}$ is the interparticle force. In the absence of tangential contact forces, this $\boldsymbol{F}$ equates to the last two terms of Eq. (5),

$$
\boldsymbol{F}=C_{2} a \eta_{s}(\dot{\boldsymbol{r}} \cdot \boldsymbol{n}) \boldsymbol{n}-C_{3} a^{2} \eta_{s} \dot{\gamma} \Theta(2 a-r) \boldsymbol{n} .
$$

We continue to use this equation even in the presence of friction. This might appear to be a drastic additional assumption but in fact our own data (generated with the DEM simulation introduced below), and also that of [27], show that in the shear thickening range of volume fractions, tangential contributions to the stress remain subdominant. This subdominance does not contradict the fact that friction, by constraining tangential particle motion, greatly enhances normal contact forces. This enhancement is captured by Eq. (8) for $C_{3}$, which diverges at a jamming point that depends on both timedependent microstructure (via $\xi$ ) and stress-dependent friction $\left(\operatorname{via} \xi^{J}\right)$.

Combining Eqs. (10), (15), (25), and (26) gives

$$
\boldsymbol{\Sigma}=\eta_{s} \alpha \boldsymbol{E}:\langle\boldsymbol{n n n n}\rangle-\eta_{s} \chi \dot{\gamma}\langle\boldsymbol{n n}\rangle_{c},
$$

where $\langle\boldsymbol{n} \boldsymbol{n}\rangle_{c}$ is the second order orientation moment of the contact part of $\Psi(\boldsymbol{r})$,

$$
\langle\boldsymbol{n n}\rangle_{c}=\int_{r=2 a}^{r=2 a+\epsilon} \Psi(\boldsymbol{r}) \Theta(2 a-r) \boldsymbol{n} \boldsymbol{n} d^{3} \boldsymbol{r} .
$$

The leading order behaviors of the prefactors $\alpha$ and $\chi$ in Eq. (27) are found from Eqs. (6)-(8) as

$$
\alpha(\phi)=\frac{\alpha_{0}}{\left(1-\phi / \phi_{1}^{J}\right)^{2}}, \quad \chi\left(\phi, \xi, \xi^{J}\right)=\frac{\chi_{0}}{\left(1-\xi / \xi^{J}\right)^{2}} .
$$

Here, $\alpha_{0}$ and $\chi_{0}$ are fitting parameters, and $\xi^{J}$ depends on the particle pressure as specified in Eq. (9).

To obtain a closed form, the contact moments $\langle\boldsymbol{n n}\rangle_{c}$ need to be approximated in terms of the coarse-grained moments $\langle\boldsymbol{n n}\rangle$, which includes all particle pairs in the coarse-graining shell. To relate $\langle\boldsymbol{n} \boldsymbol{n}\rangle_{c}$ to $\langle\boldsymbol{n} \boldsymbol{n}\rangle$, we assume the following approximate parameterization for $\Psi(\boldsymbol{r})$ within the coarse-graining shell:

$$
\begin{aligned}
\Psi(\boldsymbol{r}) & =\Psi_{\boldsymbol{n}}(\boldsymbol{n}) \Psi_{r}(r) \\
& =\Psi_{\boldsymbol{n}}(\boldsymbol{n}) a^{-2}\left[\boldsymbol{\epsilon}^{-1}-2 C_{4} \frac{\boldsymbol{E}_{c}: \boldsymbol{n} \boldsymbol{n}}{\left|\boldsymbol{E}_{c}\right|} \delta(r-2 a)\right],
\end{aligned}
$$

where $\Psi_{n}(n)$ is the orientation distribution function (with no dependence on the radial distance), $\Psi_{r}(r)$ is the radial distribution function (with no dependence on orientation), $\delta(\cdots)$ is the Dirac delta function, $C_{4}$ is a prefactor of order unity, and $\left|\boldsymbol{E}_{c}\right| \equiv \sqrt{\boldsymbol{E}_{c}: \boldsymbol{E}_{c}}$. Since closed contacts $(r=2 a)$ are predominantly oriented in a direction set by $\boldsymbol{E}_{c}$, Eq. (30) approximates the probability for closed contacts $[\Psi(r=2 a)]$ with the probability for open contacts $[\Psi(r>2 a)]$, weighted with the alignment of $\boldsymbol{n}$ in the compressive direction $-\boldsymbol{E}_{c}: \boldsymbol{n n} /\left|\boldsymbol{E}_{c}\right|$. Combining Eqs. (15) and (30), we find for 
the coarse-grained moments

$$
\begin{aligned}
\langle\boldsymbol{n n}\rangle= & \oint \Psi_{\boldsymbol{n}}(\boldsymbol{n}) \boldsymbol{n} \boldsymbol{n} d^{2} \boldsymbol{n} \\
& -C_{4} \frac{\boldsymbol{E}_{c}}{\left|\boldsymbol{E}_{c}\right|}: \oint \Psi_{\boldsymbol{n}}(\boldsymbol{n}) \boldsymbol{n n n n n} d^{2} \boldsymbol{n}
\end{aligned}
$$

Assuming $\langle\boldsymbol{n n}\rangle_{c} \ll\langle\boldsymbol{n n}\rangle$ [see Fig. 5(b)], we ignore the second term on the rhs of Eq. (31) and obtain

$$
\langle\boldsymbol{n n}\rangle=\oint \Psi_{n}(\boldsymbol{n}) \boldsymbol{n n} d^{2} n
$$

Combining Eqs. (28) and (30) gives for the contact moments,

$$
\langle\boldsymbol{n n}\rangle_{c}=-C_{4} \frac{\boldsymbol{E}_{c}}{\left|\boldsymbol{E}_{c}\right|}: \oint \Psi_{\boldsymbol{n}}(\boldsymbol{n}) \boldsymbol{n n n n n} d^{2} \boldsymbol{n} .
$$

By combining Eqs. (32) and (33), we arrive at the following relation between the contact microstructure $\langle\boldsymbol{n n}\rangle_{c}$ and the coarse-grained microstructure $\langle\boldsymbol{n n}\rangle$

$$
\langle\boldsymbol{n n}\rangle_{c}=-\frac{\boldsymbol{E}_{c}}{\left|\boldsymbol{E}_{c}\right|}:\langle\boldsymbol{n n n n n}\rangle .
$$

Here, we have set the proportionality constant $C_{4}$ to unity; Eq. (34) thus identifies an approximated, non-normalized, contact microstructure that is calculable within our coarsegrained constitutive model. Inserting Eqs. (29) and (34) into Eq. (27) gives

$$
\boldsymbol{\Sigma}=\eta_{s}\left[\frac{\alpha_{0} \boldsymbol{E}}{\left(1-\phi / \phi_{1}^{J}\right)^{2}}+\frac{\chi_{0} \boldsymbol{E}_{c}}{\left(1-\xi / \xi^{J}\right)^{2}}\right]:\langle\boldsymbol{n n n n n}\rangle,
$$

where the "jamming coordinate,"

$$
\xi=\operatorname{Tr}\langle\boldsymbol{n} \boldsymbol{n}\rangle_{c}
$$

serves as a proxy for the coordination number $Z$ for direct particle contacts. Combining Eqs. (34) and (36) gives

$$
\xi=-\frac{\langle\boldsymbol{n n}\rangle: \boldsymbol{E}_{c}}{\left|\boldsymbol{E}_{c}\right|}
$$

Without a relation such as Eq. (34), the distance from jamming is not deducible from the coarse-grained microstructure tensor $\langle\boldsymbol{n n}\rangle$ : a proxy of some sort is essential for our constitutive model of shear thickening to be closed at a coarsegrained level. However, Eq. (37) comprises a relatively crude approximation; some other combination of $\langle\boldsymbol{n n}\rangle$ and flow tensors might approximate $Z$ more accurately. Indeed, it is found in particle-based simulations that the reduced viscosity $\eta_{r}$ in steady shear flow has a different power-law dependence on each: $\left(1-\xi / \xi^{J}\right)^{-2} \sim \eta_{r} \sim\left(1-Z / Z^{J}\right)^{-4}$ [14]. Moreover, we will see in Fig. 4 below that $\langle\boldsymbol{n n}\rangle_{c}$ has some shortcomings when compared with the results of particle-based simulations.

With this in mind, although $\xi$ was constructed above as an estimator of $Z$, we note that its conceptual role in our constitutive model does not require this interpretation. Instead, it can be viewed as a microstructural scalar that can capture the distance from a jamming point, $\xi-\xi^{J}$, in time-dependent flows, just as $\phi-\phi^{J}$ does in the WC theory for steady flow [14]. The jamming coordinate $\xi$ thereby emerges as the central variable to model shear thickening: in Eq. (9), the stress is assumed to diverge when $\xi$ reaches a critical value $\xi^{J}$, that smoothly reduces from a larger frictionless value $\xi_{1}^{J}$, to a smaller frictional value $\xi_{2}^{J}$, when the pressure $\Pi$ in the system exceeds the onset value $\Pi^{*}$.

\section{Determination of parameters}

The critical values $\xi_{1}^{J}$ and $\xi_{2}^{J}$ are found by demanding that in steady shear frictionless and frictional jamming occur at volume fractions $\phi_{1}^{J}$ and $\phi_{2}^{J}$, respectively. For steady $x y$ shear flow, the solution to Eq. (24) reads

$$
\begin{aligned}
\langle\boldsymbol{n n}\rangle= & \frac{\phi}{\left(9 \beta^{2}+54 \beta+416\right)} \\
& \times\left\{\frac{1}{15}\left(129 \beta^{2}-374 \beta+3256\right) \boldsymbol{\delta}_{1} \boldsymbol{\delta}_{1}\right. \\
& -\frac{28}{5}\left(\beta^{2}-3 \beta\right)\left(\boldsymbol{\delta}_{1} \boldsymbol{\delta}_{2}+\boldsymbol{\delta}_{2} \boldsymbol{\delta}_{1}\right) \\
& +\frac{1}{15}\left(129 \beta^{2}+410 \beta+904\right) \boldsymbol{\delta}_{2} \boldsymbol{\delta}_{2} \\
& \left.+\frac{1}{15}\left(87 \beta^{2}+564 \beta+820\right) \boldsymbol{\delta}_{3} \boldsymbol{\delta}_{3}\right\},
\end{aligned}
$$

where we recall that $\beta$ depends on $\phi$ [Eq. (21)]. Inserting Eq. (38) into Eq. (37) gives for the jamming coordinate in steady shear,

$$
\xi=\phi \frac{\left(213 \beta^{2}-234 \beta+2080\right)}{15\left(9 \beta^{2}+54 \beta+416\right)}
$$

The factor $\phi$ in Eq. (39) follows from the assumption [Eq. (23)] that the outer distribution of the pair separation vector is proportional to $\phi$.

Requiring that frictionless and frictional jamming occur at volume fractions $\phi_{1}^{J}$ and $\phi_{2}^{J}$ demands the following critical values for the frictionless and frictional jamming coordinates:

$$
\xi_{1,2}^{J}=\phi_{1,2}^{J} \frac{\left(213 \beta^{2}-234 \beta+2080\right)}{15\left(9 \beta^{2}+54 \beta+416\right)} .
$$

Equations (9), (21), (24), (29), (35), (37), and (40) form a closed system for the microstructure and stress. For any given volume fraction $\phi$, the model contains parameters $\alpha_{0}, \beta_{0}, \chi_{0}$, $\Pi^{*}, \phi_{1}^{J}$ and $\phi_{2}^{J}$. Of these parameters, $\phi_{1}^{J}$ and $\phi_{2}^{J}$ are directly determinable from experimental or computational data pertaining the dependence of the viscosity on the volume fraction under frictionless and frictional conditions, respectively, and $\Pi^{*}$ enters only through the scale factor relating the reduced shear rate $\dot{\gamma}_{r}$ [Eq. (1)] to the actual one, $\dot{\gamma}$. In previous work, we used steady state microstructural and viscosity data (for various $\phi$ ), and microstructural reversal data (for $\phi=0.56)$, from particle-based simulations, to fit 
$\phi_{1}^{J}=0.65, \phi_{2}^{J}=0.57, \Pi^{*}=0.037 F^{*} / a^{2}, \alpha=120, \beta=50$, and $\chi_{0}=2.4$, which for $\phi=0.56$ corresponds to $\alpha_{0}=2.3$ and $\beta_{0}=6.9$. It is noted that these $\phi_{1,2}^{J}$ differ slightly from the values extrapolated from the DEM data $\phi_{1}^{J}=0.644, \phi_{2}^{J}=0.578$. In [14], the model was then used to predict, without further parameter fitting, the rheological reversal data at $\phi=0.56$, with qualitatively good agreement in most respects [14]. It is also noted that according to Eq. $(40), \xi_{1,2}^{J} \approx 0.88,0.78$, while the corresponding coordination numbers are $Z_{1,2}^{J}=6,4$. It is, therefore, re-emphasized that, although $\xi$ might be interpreted as an approximation for $Z$, these parameters differ numerically, and they are not linearly proportional. They nevertheless play similar roles, in providing the distance to the jamming point.

We next briefly review the particle-based simulation methodology before making a similar comparison of the constitutive model with a contrasting type of flow, in which transverse oscillations are superposed onto steady shearing.

\section{DISCRETE-ELEMENT MODEL}

Our DEM simulation model considers non-Brownian, almost noninertial, neutrally buoyant particles in a periodic cubic box at volume fraction $\phi$. The particles are an equimolar mixture of radii $a$ and $1.4 a$, and have density $\rho$. The box is initialized with 1500 nonoverlapping particles, and we report averages over 10 realizations. The simulation box (volume $V$ ) is deformed with a superposition of a steady shear flow (rate $\dot{\gamma}$ ) and a transverse oscillating shear flow (amplitude $\gamma$ and frequency $\omega$ ) with a velocity gradient $\boldsymbol{L}$ and rate of strain tensor $\boldsymbol{E}$ that are given by Eqs. (45) and (46) below, respectively. The nondimensional control parameters for this family of flows are, when applied to shear-thickening suspensions, the volume fraction $\phi$, the oscillation strain amplitude $\gamma$, the dimensionless oscillation frequency $\dot{\gamma}_{\perp}$ [Eq. (4)] and the dimensionless shear rate $\dot{\gamma}_{r}$ [Eq. (1)]. For the transverse flow to be effective at reducing the viscosity, its amplitude must be large enough to break direct contacts yet small enough to inhibit significant contact formation in $y z$. Within this range (approximately $10^{-4}<\gamma<0.05$ ), the results are almost independent of $\gamma$ [21], and in the following, we fix $\gamma=0.01$.

Hydrodynamic interactions between particles are computed as described in [26] and [29-31]. For neighboring particles 1 and 2, translating with velocities $\boldsymbol{U}_{1}, \boldsymbol{U}_{2}$ and rotating at $\boldsymbol{\Omega}_{1}, \boldsymbol{\Omega}_{2}$, and with center-center vector $\boldsymbol{r}$ (and $\boldsymbol{n}=\boldsymbol{r} /|\boldsymbol{r}|$ ) pointing from particle 2 to particle 1, the force $\boldsymbol{F}^{h}$ and torque $\boldsymbol{\Gamma}^{h}$ on particle 1 are given by

$$
\begin{gathered}
\boldsymbol{F}^{h} / \eta_{s}=\left[X_{11}^{A} \boldsymbol{n n}+Y_{11}^{A}(\boldsymbol{\delta}-\boldsymbol{n n})\right] \cdot\left(\boldsymbol{U}_{2}-\boldsymbol{U}_{1}\right) \\
+Y_{11}^{B}\left(\boldsymbol{\Omega}_{1} \times \boldsymbol{n}\right)+Y_{21}^{B}\left(\boldsymbol{\Omega}_{2} \times \boldsymbol{n}\right), \\
\boldsymbol{\Gamma}^{h} / \eta_{s}=Y_{11}^{B}\left(\boldsymbol{U}_{2}-\boldsymbol{U}_{1}\right) \times \boldsymbol{n}-(\boldsymbol{\delta}-\boldsymbol{n n}) \cdot\left(Y_{11}^{C} \boldsymbol{\Omega}_{1}+Y_{12}^{C} \boldsymbol{\Omega}_{2}\right),
\end{gathered}
$$

where $\eta_{s}$ is the solvent viscosity. The surface-surface separation is given, for particle radii $a_{1}$ and $a_{2}$, by $h=|\boldsymbol{r}|-\left(a_{1}+a_{2}\right)$, which is nondimensionalized as $2 h /\left(a_{1}+a_{2}\right)$. The scalar resistances $X_{11}^{A}, Y_{11}^{A}, Y_{11}^{B}, Y_{21}^{B}, Y_{11}^{C}$, and $Y_{12}^{C}$ are given elsewhere [32]. We neglect interactions that have $h>0.05 a$. A drag force and torque act on particle 1 at position $\boldsymbol{x}_{1}$, given by

$$
\begin{aligned}
& \boldsymbol{F}^{d}=-6 \pi \eta_{s} a_{1}\left(\boldsymbol{U}_{1}-\boldsymbol{U}\left(\boldsymbol{x}_{1}\right)\right), \\
& \boldsymbol{\Gamma}^{d}=-8 \pi \eta_{s} a_{1}^{3}\left(\boldsymbol{\Omega}_{1}-\boldsymbol{\Omega}\left(\boldsymbol{x}_{1}\right)\right),
\end{aligned}
$$

with $\boldsymbol{\Omega}=\frac{1}{2} \nabla \times \boldsymbol{U}$ being the fluid vorticity vector, and the streaming velocity is given by $\boldsymbol{U}(\boldsymbol{x})=\boldsymbol{L} \cdot \boldsymbol{x}$.

Below a separation $h_{\min }=0.001 a$, hydrodynamic forces are regularized and particles enter into direct contact. Particle pairs with overlap $\delta=\left(\left(a_{1}+a_{2}\right)-|\boldsymbol{r}|\right) \Theta\left(\left(a_{1}+a_{2}\right)-|\boldsymbol{r}|\right)$ (with Heaviside function $\Theta$ ) and center-center unit vector $\boldsymbol{n}$ lead to contact force and torque on particle 1 according to [33]

$$
\boldsymbol{F}^{c}=k_{n} \delta \boldsymbol{n}-k_{t} \boldsymbol{t}
$$

$$
\Gamma^{c}=a_{1} k_{t}(\boldsymbol{n} \times \boldsymbol{t}),
$$

where $\boldsymbol{t}$ represents the incremental tangential displacement, reset at the initiation of each contact. Here, $k_{n}$ and $k_{t}$ are stiffnesses, with $k_{t}=(2 / 7) k_{n}$. The tangential force component is restricted by a friction coefficient $\mu=1$ so that $\left|k_{t} t\right| \leq \mu k_{n} \delta$. Stress-dependence enters through $\mu$, following [1],

$$
\left|k_{t} t\right| \leq \begin{cases}\mu k_{n}\left(\delta-\delta^{*}\right) & \text { for } \delta>\delta^{*} \\ 0 & \text { otherwise }\end{cases}
$$

where $F^{*} \equiv k_{n} \delta^{*}$ is the normal force above which friction is activated, leading to a nondimensional shear rate $\dot{\gamma}_{r}=\dot{\gamma} \eta_{s} / \Pi^{*} \sim \dot{\gamma} \eta_{s} a^{2} / F^{*}$.

Particle trajectories are computed from the above forces, and the components of the stress tensor $\boldsymbol{\Sigma}$ are calculated by summing $-\boldsymbol{F r}$ over all interacting particle pairs and dividing by $V$. The contact microstructure is computed as $\langle\boldsymbol{n} \boldsymbol{n}\rangle_{c}$, where $\langle\cdots\rangle_{c}$ denotes averaging over all particle pairs for which the contact forces [Eq. (43a)] are activated. We also construct a coarse-grained microstructure $\langle\boldsymbol{n n}\rangle$, where $\langle\cdots\rangle$ averages over all particle pairs that interact via direct contact forces or lubrication forces, the latter being cut-off beyond a separation distance of $h=0.05 a$. Below, we will compare these quantities to constitutive model predictions. In addition to the control parameters described above, the model leads to a Stokes number $\mathrm{St}=\rho \dot{\gamma} a^{2} / \eta_{s}$ and a $k_{n}$-scaled shear rate $\hat{\dot{\gamma}}=2 \dot{\gamma} a / \sqrt{k_{n} /(2 \rho a)}$. We set St $<10^{-3}$ and $\hat{\dot{\gamma}}<10^{-5}$ to approximate inertia-free, hard sphere conditions. The model is implemented in LAMMPS [34].

\section{RESULTS}

We now test the microstructure and stress predicted by our constitutive model against data generated by the DEM simulation at volume fraction $\phi=0.56$, under a homogeneous, 
time-dependent velocity gradient,

$$
\boldsymbol{L}=\left(\begin{array}{ccc}
0 & \dot{\gamma} & 0 \\
0 & 0 & 0 \\
0 & \omega \gamma \cos (\omega t) & 0
\end{array}\right)
$$

corresponding to a deformation rate

$$
\mathbf{E}=\left(\begin{array}{ccc}
0 & \frac{1}{2} \dot{\gamma} & 0 \\
\frac{1}{2} \dot{\gamma} & 0 & \frac{1}{2} \gamma \omega \cos (\omega t) \\
0 & \frac{1}{2} \gamma \omega \cos (\omega t) & 0
\end{array}\right) .
$$

In the limit of large $\dot{\gamma}_{\perp}$, we have that

$$
\boldsymbol{E}_{e}=\frac{1}{2} \gamma \omega|\cos (\omega t)| \boldsymbol{n}_{e} \boldsymbol{n}_{e}, \quad \boldsymbol{E}_{c}=-\frac{1}{2} \gamma \omega|\cos (\omega t)| \boldsymbol{n}_{c} \boldsymbol{n}_{c},
$$

where $\pm \frac{1}{2} \gamma \omega|\cos (\omega t)|$ are the expansive and compressive eigenvalues of $\boldsymbol{E}$ of Eq. (46) and $\boldsymbol{n}_{e}=x(0,1,1) / \sqrt{2}+$ $(1-x)(0,-1,1) / \sqrt{2} \quad$ and $\quad \boldsymbol{n}_{c}=(1-x)(0,1,1) / \sqrt{2}+$ $x(0,-1,1) / \sqrt{2}$ are the corresponding eigenvectors, with $x=\Theta[\cos (\omega t)]$ and $\Theta(\cdot)$ is the Heaviside step function. Note that these eigenvectors interchange their direction after each half oscillation period and, on average, $-\bar{E}_{c}=\bar{E}_{e} \sim \gamma \omega$ $\left(\boldsymbol{\delta}_{y} \boldsymbol{\delta}_{y}+\boldsymbol{\delta}_{z} \boldsymbol{\delta}_{z}\right)$.

Setting $\gamma=0.01$ and $\phi=0.56$, the remaining control parameters are $\dot{\gamma}_{r}$ [Eq. (1)] and $\dot{\gamma}_{\perp}$ [Eq. (4)] which quantify the influence, respectively, of frictional contact forces and transverse oscillations. Below, we first focus on the limiting cases of $\dot{\gamma}_{\perp}=0$ and $\dot{\gamma}_{\perp}=\infty$, before considering the behavior of shear-thickened suspensions $\left(\dot{\gamma}_{r} \gg 1\right)$ at intermediate values of $\dot{\gamma}_{\perp}$. We finally present full maps of the viscosity as functions of $\dot{\gamma}_{r}$ and $\dot{\gamma}_{\perp}$.

\section{A. Steady behavior with $\dot{\gamma}_{\perp}=\mathbf{0}$}

A flow curve for steady shear without transverse oscillation $\left(\dot{\gamma}_{\perp}=0\right)$ is shown in Fig. 2(a), demonstrating good agreement in the viscosity prediction of the DEM simulation and the constitutive model. The parameter values are those chosen in [14] as detailed in Sec. II D. The constitutive model predicts for the shear component of the coarse-grained microstructure that $\langle\boldsymbol{n n}\rangle_{x y}<0$ and for the normal components that $\langle\boldsymbol{n n}\rangle_{y y}>\langle\boldsymbol{n} \boldsymbol{n}\rangle_{x x}>\langle\boldsymbol{n n}\rangle_{z z}$, and similar behavior for the contact microstructure $\langle\boldsymbol{n n}\rangle_{c, i j}$. The model thus predicts a
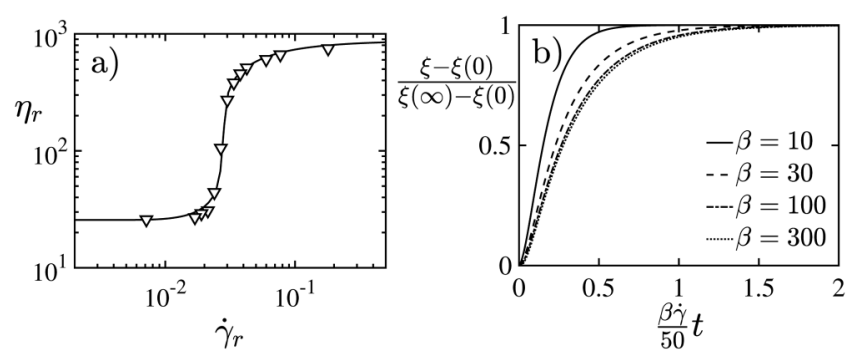

FIG. 2. (a) Relative suspension viscosity $\eta_{r}$ in steady shear as a function of dimensionless shear rate $\dot{\gamma}_{r}$, measured in the absence of transverse oscillations, $\dot{\gamma}_{\perp}=0$. Shown are results from the constitutive model (solid line) and discrete-element simulation (triangles) at $\phi=0.56$. (b) Recovery of the jamming coordinate $\xi$ after shear reversal scaled with its values at $t=0$ and at $t=\infty$, for various values of the microstructure association rate $\beta$. positive first normal stress difference $\zeta_{1}=\left(\Sigma_{x x}-\Sigma_{y y}\right) / \Sigma_{x y}$ and a negative second normal stress difference $\zeta_{2}=\left(\Sigma_{y y}-\Sigma_{z z}\right) / \Sigma_{x y}$. This is in partial agreement with DEM, which predicts that $\langle\boldsymbol{n n}\rangle_{x y}<0$ and that $\langle\boldsymbol{n n}\rangle_{x x}>\langle\boldsymbol{n n}\rangle_{y y}$ $>\langle\boldsymbol{n n}\rangle_{z z}$, and similar behavior for $\langle\boldsymbol{n n}\rangle_{c, i j}$. Correspondingly, DEM predicts that $\zeta_{1}<0$ (but very small) and $\zeta_{2}<0$. In general, the constitutive model overestimates the microstructural anisotropy and $\left|\zeta_{1,2}\right|$, as compared to the DEM simulation [14]. Further results and discussion relating to the steady shear stress and the microstructure predicted by our model are given in [14].

\section{B. Shear reversal with $\dot{\gamma}_{\perp}=0$}

Figure 2(b) shows the recovery of the jamming coordinate $\xi$ [Eq. (37)] after shear reversal for various values of the microstructure association rate $\beta$. In this case, the suspension is subjected to a negative $x y$-shear flow, without $z y$-shear oscillations. When the steady state is reached, the shear flow is reversed at $t=0$. It is seen that, for $\beta \gtrsim 30$, the microstructural recovery rate $\approx \beta \dot{\gamma} / 50$ and full recovery is achieved after a strain of $\approx 50 / \beta$. This suggests that, in the constitutive model, the (transverse) oscillatory strain $\gamma=10^{-2}$ is unable to induce significant microstructural reorganization, for the present case, where $\beta=50$.

\section{Limiting behavior for large $\dot{\gamma}_{\perp}$}

Next, we consider $\langle\boldsymbol{n} \boldsymbol{n}\rangle$ predicted by the constitutive model, in the limit of a very large oscillation frequency $\dot{\gamma}_{\perp} \gg 1$. In this limit, $\langle\boldsymbol{n} \boldsymbol{n}\rangle$ is dominated by the oscillatory flow, while the steady shear component only presents an $\mathcal{O}\left(\dot{\gamma}_{\perp}^{-1}\right)$ perturbation to $\langle\boldsymbol{n n}\rangle$. We have seen in Fig. 2(b) that substantial microstructural reorganization requires $\beta \gamma / 50 \gtrsim 1$, which is not met by current conditions, in which $\beta=50$ and $\gamma=10^{-2}$. Under present conditions, the periodic changes in $\langle\boldsymbol{n n}\rangle$ are $\mathcal{O}(\beta \gamma / 50)$, while on average, $\langle\boldsymbol{n n}\rangle$ remains isotropic and equilibrated to the external microstructure $\langle\boldsymbol{n} \boldsymbol{n}\rangle=\langle\boldsymbol{n} \boldsymbol{n}\rangle^{\text {outer }}+\mathcal{O}(\beta \gamma / 50)$, where $\langle\boldsymbol{n} \boldsymbol{n}\rangle^{\text {outer }}=\phi \boldsymbol{\delta} / 3$.

The nearly isotropic $\langle\boldsymbol{n n}\rangle$ at $\dot{\gamma}_{\perp} \gg 1$ corresponds to a jamming coordinate of $\xi \approx \phi / 3$ [found by inserting $\langle\boldsymbol{n n}\rangle=\phi \boldsymbol{\delta} / 3$ in Eq. (37)], roughly four times smaller than $\xi \approx 1.4 \phi$, which follows from inserting the steady shear microstructure [Eq. (38)] into Eq. (37) and using our chosen model parameter $\beta=50$.

If one now imposes a small steady shear flow perpendicular to this oscillatory state, the ability to flow in the steady direction is governed by the time-averaged $\langle\boldsymbol{n n}\rangle$, which is isotropized by the dominant oscillatory flow. This isotropization corresponds to a lower $\xi \approx \phi / 3$ as compared to that in steady shear $\xi \approx 1.4 \phi$, taking the system further from jamming, thereby causing a reduction in the modeled stress, via Eq. (35); for a system close to the steady-shear jamming point, this reduction can be arbitrarily large. This explanation of the unjamming effect of transverse oscillation in the large $\dot{\gamma}_{\perp}$ limit is broadly consistent with previous discussions [21] and [22].

In what follows, we solve the full constitutive model numerically, across a wide range of $\dot{\gamma}_{\perp}$. We thereby confirm that for very large $\dot{\gamma}_{\perp}$, the model predicts an isotropic 
coarse-grained microstructure $\langle\boldsymbol{n n}\rangle \approx \phi \boldsymbol{\delta} / 3 \quad$ [Fig. 5(c)] with $\xi \approx \phi / 3$.

\section{Role of $\dot{\gamma}_{\perp}$ : Transient behavior}

We next compare results for intermediate values of $\dot{\gamma}_{\perp}$, focusing again on the shear-thickened case, $\Pi \gg \Pi^{*}$. This case is described by the limit $\dot{\gamma}_{r}=\infty$ where frictional contacts are maximized $\left[\Pi^{*}=0\right.$ and $f=1$ in Eq. (9)] so that the role of particle-particle contact forces, at least under steady shear flow, is maximally important. We first present the behavior observed in discrete-element simulations before discussing the constitutive model predictions.

Shown in Figs. 3(a) and 3(c) are examples of time series for the steady shear stress $\Sigma_{x y}$, and the transverse one $\Sigma_{y z}$, as well as the corresponding components of the contact microstructure tensor, $\langle\boldsymbol{n} \boldsymbol{n}\rangle_{c, x y}$ and $\langle\boldsymbol{n n}\rangle_{c, y z}$, as obtained by DEM simulations with $\dot{\gamma}_{r}=\infty$ and $\dot{\gamma}_{\perp}=0.2$. Starting from a contact-free state, the steady shear flow component leads to a gradual building of particle contacts, predominantly oriented along the compressive direction of the steady shear. This process results in a large shear stress $\Sigma_{x y}$ and a negative contact microstructure component $\langle\boldsymbol{n n}\rangle_{c, x y}$. (Note that the definition of $\langle\boldsymbol{n n}\rangle_{c, x y}$ is such that it is negative under $x y$-shear flow with positive $\partial_{y} u_{x}$.) Meanwhile, the transverse shear generates oscillations in $\Sigma_{y z}$ in phase with the oscillatory shear rate $\gamma \omega \cos (\omega t)$, whose amplitude increases during the first few cycles as the steady flow component generates contacts. The transverse component of the contact microstructure $\langle\boldsymbol{n n n}\rangle_{c, y z}$ oscillates in antiphase with the oscillatory shear rate, which is understood by noting that, at $\dot{\gamma}_{\perp}=0.2$, the microstructural response is sufficiently fast compared to the change in flow direction that we essentially have a series of steady-state shear flows with a slowly changing direction [21,22].

In Figs. 3(b) and 3(d), we show time series of the stress components $\Sigma_{x y}, \Sigma_{y z}$ and of the contact microstructure
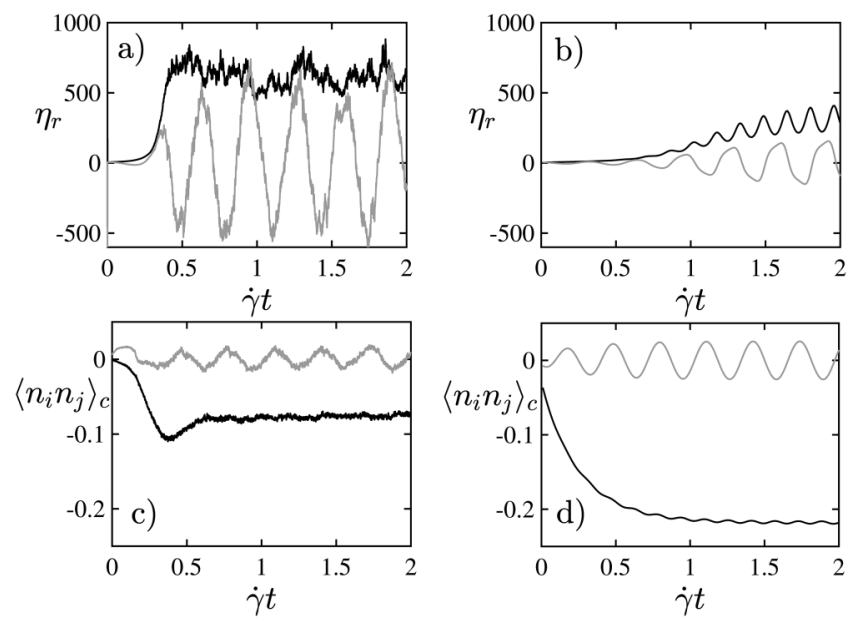

FIG. 3. Transient response of DEM simulation and constitutive model to shear flow with superposed transverse oscillations for $\dot{\gamma}_{r}=\infty$ and $\dot{\gamma}_{\perp}=0.2$. Shown are steady shear viscosity $\Sigma_{x y} /\left(\dot{\gamma} \eta_{s}\right)$ (black) and oscillatory viscosity component $\Sigma_{y z} /\left(\omega \gamma \eta_{s}\right)$ (gray) for DEM (a) and constitutive model (b), and contact microstructure components $\langle\boldsymbol{n n}\rangle_{c, x y}$ (black) and $\langle\boldsymbol{n n}\rangle_{c, y z}$ (gray) for DEM (c) and constitutive model (d). components $\langle\boldsymbol{n n}\rangle_{c, x y}$ and $\langle\boldsymbol{n n}\rangle_{c, y z}$, predicted by the constitutive model with $\dot{\gamma}_{r}=\infty$ and $\dot{\gamma}_{\perp}=0.2$. The constitutive model agrees qualitatively with the DEM [Figs. 3(a) and 3(c)]. The contact microstructure $\langle\boldsymbol{n n}\rangle_{c, x y}$ develops over a few steady strain units, accompanied by substantial growth of $\Sigma_{x y}$ and $\Sigma_{y z}$. After the initial transient, $\langle\boldsymbol{n n}\rangle_{c, x y}$ is nearly steady and negative, while $\langle\boldsymbol{n n}\rangle_{c, y z}$ oscillates in antiphase to the transverse shear rate $\gamma \omega \cos (\omega t)$. Quantitatively, the constitutive model requires a larger strain for $\Sigma_{x y}$ to develop fully, and it does not capture the transient peak in $\langle\boldsymbol{n n}\rangle_{c, x y}$.

However, while the transverse viscosity response found by DEM simulation remains in phase with the transverse shear rate for all $\dot{\gamma}_{\perp}$, the phase angle of $\langle\boldsymbol{n n n}\rangle_{c, y z}$ shows a nonmonotonic dependence on $\dot{\gamma}_{\perp}$. Shown in Fig. 4 are the phase angle $\theta$ of $\langle\boldsymbol{n n n}\rangle_{c, y z}$ relative to minus the $z y$-strain $[-\gamma \sin (\omega t)]$ found by DEM simulation and the same phase angle for both $\langle\boldsymbol{n} \boldsymbol{n}\rangle$ and $\langle\boldsymbol{n n}\rangle_{c}$ in the constitutive model. The DEM simulations show that $\theta$ transitions as a function of $\dot{\gamma}_{\perp}$ from (i) $\theta \approx \pi / 2$ (antiphase with the $z y$-shear rate), via (ii) $\theta \approx \pi$ (in-phase with the $z y$-strain), to (iii) $\theta \approx 0$ (antiphase with the $z y$-strain). The physics of this sequence is explored in detail elsewhere [21]. Briefly, the three regimes correspond to (i) instant adaptation, where the contact microstructure tensor tracks the velocity gradient tensor as this oscillates around its mean value in a quasisteady-state fashion; (ii) chain tilting, where the oscillatory flow deforms contacts faster than they are replaced by new ones but does not break up force chains; and (iii) chain breaking where the flowinduced contact network of the steady shear is substantially disrupted by the transverse oscillation.

The constitutive model predicts different behaviors of the phase angle depending on whether the contact microstructure $\langle\boldsymbol{n n}\rangle_{c}$ or the coarse-grained microstructure $\langle\boldsymbol{n} \boldsymbol{n}\rangle$ is considered. The first of these shows $\theta \approx \pi / 2$ over the entire $\dot{\gamma}_{\perp}$-range and is quite unlike the DEM data. Interestingly, this discrepancy is inherent in the definition of $\langle\boldsymbol{n} \boldsymbol{n}\rangle_{c}$ in Eq. (34). It follows from this definition that the oscillations in $\langle\boldsymbol{n} \boldsymbol{n}\rangle_{c}$ must remain almost in antiphase with the oscillations in $\boldsymbol{E}$. This is readily seen in the limit $\dot{\gamma}_{\perp}=\infty$, where $\boldsymbol{E}_{c}=E_{c, y z}\left(\boldsymbol{\delta}_{y} \boldsymbol{\delta}_{z}+\boldsymbol{\delta}_{z} \boldsymbol{\delta}_{y}\right)$ since $E_{c, x y} / E_{c, y z} \sim \dot{\gamma}_{\perp}^{-1}=0$, and $\langle\boldsymbol{n n}\rangle \approx \boldsymbol{\phi} \boldsymbol{\delta} / 3$ (see Sec. IV C). Inserting these expressions and Eq. (22) into Eq. (34) gives $\langle\boldsymbol{n n}\rangle_{c, y z}=-(\phi / 15)$ $E_{c, y z} /\left|E_{c, y z}\right|$, which is in antiphase with $E_{c, y z}$. This phase discrepancy shows that improvement of our ansatz Eq. (34) for

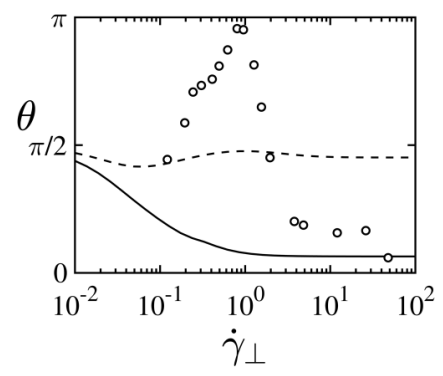

FIG. 4. Phase angle $\theta$ for the coarse-grained microstructure component $\langle\boldsymbol{n n}\rangle_{y z}$ in the constitutive model (solid line), for the contact microstructure component $\langle\boldsymbol{n n}\rangle_{c, y z}$ in the constitutive model (dashed line) and for the contact microstructure component $\langle\boldsymbol{n n}\rangle_{c, y z}$ in DEM simulation (markers). 
the contact microstructure should be a priority for future refinement of our constitutive model.

The oscillations of the coarse-grained microstructure tensor $\langle\boldsymbol{n n}\rangle$, on the other hand, are not enslaved to those of $\boldsymbol{E}$. As a result, the phase angle for the coarse-grained microstructure $\langle\boldsymbol{n n}\rangle$ evolves in better qualitative agreement with the contact microstructure $\langle\boldsymbol{n n}\rangle_{c}$ found from the DEM simulations. Figure 4 shows that with increasing $\dot{\gamma}_{\perp}$, the corresponding phase angle transitions smoothly from $\theta \approx \pi / 2$ (antiphase with the $z y$-shear rate) to $\theta \approx 0$ (antiphase with the $z y$-strain). The transition in the constitutive model occurs when the oscillation frequency $\omega$ exceeds the microstructure formation rate $\dot{\gamma} \beta / 50$ [see Fig. 2(b)], which corresponds to $\dot{\gamma}_{\perp}=\beta \gamma / 50=10^{-2}$. In the classification of [21], the model seemingly captures both regime (i) instant adaptation and regime (iii) chain breaking. However, the peak in the phase angle plot at $\dot{\gamma}_{\perp} \approx 1$, which corresponds to the chain-tilting regime (ii), is notably absent from the prediction of our constitutive model. This regime is characterized by a pseudoelastic microstructural response, while the stress response itself remains viscous. (See Sec. V for further discussion.)

\section{E. Role of $\dot{\gamma}_{\perp}$ : Time-averaged response}

We next present the viscosity and the microstructure, as predicted by the DEM simulation and the constitutive model, averaged over the oscillation cycle, again focusing on the fully shear-thickened case with $\dot{\gamma}_{r}=\infty$.

The constitutive model qualitatively predicts the $\dot{\gamma}_{\perp}$-mediated decrease in suspension viscosity $\eta_{r}=\Sigma_{x y} /\left(\dot{\gamma} \eta_{s}\right)$, Fig. 5(a), consistent with our DEM simulation data and

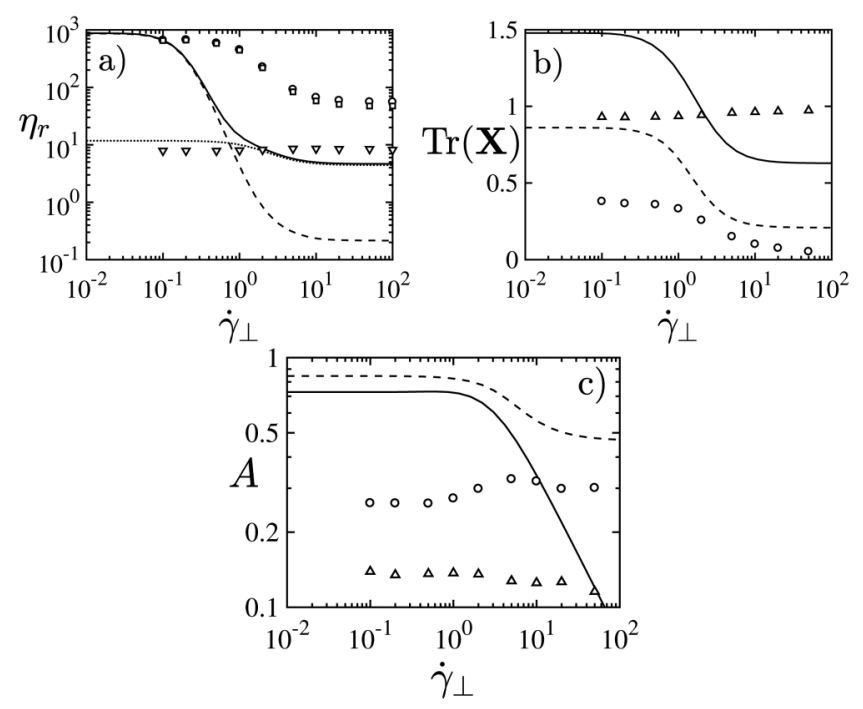

FIG. 5. Time-averaged response of constitutive model and DEM simulation to shear flow with superposed transverse oscillations. (a) Total suspension viscosity $\Sigma_{x y} / \dot{\gamma} \eta_{s}$ (circles and solid line), contact contribution to viscosity (squares and dashed line) and hydrodynamic contribution to viscosity (triangles and dotted line) in constitutive model (lines) and DEM (markers). (b) Number of coarse-grained interactions $\operatorname{Tr}\langle\boldsymbol{n n}\rangle$ (triangles and solid line) and contact interactions $\operatorname{Tr}\langle\boldsymbol{n n}\rangle_{c}$ (circles and dashed line) normalized by $\xi_{1}^{J}$ [Eq. (40)] in constitutive model (lines), and normalized by $Z_{1}^{J}=6$ in DEM (markers). (c) Anisotropy $A$ [Eq. (48)] of the coarse-grained microstructure $\boldsymbol{X}=\langle\boldsymbol{n n}\rangle$ (triangles and solid line) and contact microstructure $\boldsymbol{X}=\langle\boldsymbol{n} \boldsymbol{n}\rangle_{c}$ (circles and dashed line), in constitutive model (lines) and DEM (markers). with experimental data measured under equivalent shearing conditions [21] and indeed under acoustic perturbations [24]. Quantitatively, however, the DEM simulations show a decrease in the viscosity by a factor of around 20 at this volume fraction (in an earlier article, we showed within DEM the dependence of this decrease on $\phi$ [22]), whereas the constitutive model predicts a drop by a factor of around 200.

This difference reflects that the contact stress in the constitutive model depends too strongly on the number of contacts [Eq. (8)]. The main discrepancy is that while the constitutive model predicts for large $\dot{\gamma}_{\perp}$ a complete collapse of the contact contribution leaving only the lubrication part, the DEM data show that the stress remains contact-dominated even at large $\dot{\gamma}_{\perp}$. Although in this regime (the chain-breaking regime of [21]) the microstructure is severely disrupted, in the DEM simulations, direct contacts are not so diminished as to contribute negligibly to stress, as the constitutive model predicts. This is due in part to the chosen operating condition of $\phi=0.56$ and $\mu=1$. Close to $\phi_{2}^{J}$, even small numbers of frictional contacts are sufficient to give a dominant contact stress. At lower $\phi$ and $\mu$, the DEM simulation does indeed predict hydrodynamic stress dominance at large $\dot{\gamma}_{\perp}$ [22].

Shown in Fig. 5(b) are the number of contact interactions $\operatorname{Tr}\langle\boldsymbol{n n}\rangle_{c}$ and the number of coarse-grained interactions $\operatorname{Tr}\langle\boldsymbol{n n}\rangle$, as functions of $\dot{\gamma}_{\perp}$ predicted by the DEM simulation and the constitutive model. For the DEM simulations, $\langle\boldsymbol{n n}\rangle_{c}$ includes direct contacts only [those for which we compute Eq. (43a)], while $\langle\boldsymbol{n n}\rangle$ corresponds to all (direct and lubricated) interactions within the lubrication cutoff length $h=0.05 a . \operatorname{Tr}\langle\boldsymbol{n n}\rangle_{c}$ decreases steadily with increasing $\dot{\gamma}_{\perp}$ as the oscillations increasingly break up force chains created by the steady shearing flow. $\operatorname{Tr}\langle\boldsymbol{n n}\rangle$, on the other hand, is only weakly affected by the transverse oscillations. This is due to the low strain amplitude, $\gamma=0.01$, which is sufficient to move particles out of direct contact, but not to move interacting particles out of each other's lubrication films (as cut-off at $h=0.05 a$ ). This result is independent of $\gamma$, provided $\gamma$ remains within the range mentioned above (approximately $\left.10^{-4}<\gamma<0.05\right)$. Overall, there is qualitative agreement in $\operatorname{Tr}\langle\boldsymbol{n n}\rangle_{c}$ between the constitutive model and discrete-element simulation, with both predicting a similar $\dot{\gamma}_{\perp}$-dependence. For $\operatorname{Tr}\langle\boldsymbol{n n}\rangle$ on the other hand, there is disagreement, where the constitutive model predicts a decrease, and the DEM predicts a constant as a function of $\dot{\gamma}_{\perp}$.

Figure 5(c) shows the time-averaged microstructural anisotropy, defined as

$$
A=\{1-27 \operatorname{Det}[\boldsymbol{X} / \operatorname{Tr}(\boldsymbol{X})]\}^{\frac{1}{3}},
$$

for the contact microstructure $\boldsymbol{X}=\langle\boldsymbol{n n}\rangle_{c}$ and for the coarsegrained microstructure $\boldsymbol{X}=\langle\boldsymbol{n n}\rangle$, both in the constitutive model and in DEM. In DEM, $\langle\boldsymbol{n n}\rangle_{c}$ has $A \approx 0.3$ and $\langle\boldsymbol{n n}\rangle$ has $A \approx 0.1$. In the constitutive model, the microstructure is more anisotropic with $A \approx 0.8$, for $\langle\boldsymbol{n n}\rangle_{c}$ and $A \approx 0.7$, for $\langle\boldsymbol{n n}\rangle$, until $\dot{\gamma}_{\perp}$ becomes large. This over-prediction of microstructural anisotropy within our constitutive model has previously been reported for steady flows [14].

When $\dot{\gamma}_{\perp}$ does become large, the constitutive model predicts near isotropization of the coarse-grained microstructure, 
$A=\mathcal{O}(\beta \gamma / 50)$, and saturating anisotropy of the contact microstructure, $A \approx 0.5 \phi$, which is found by inserting $(1 / 2)\left(\boldsymbol{\delta}_{y} \boldsymbol{\delta}_{y}+\boldsymbol{\delta}_{z} \boldsymbol{\delta}_{z}\right)$ for the time averaged value for $-\boldsymbol{E}_{c} /\left|\boldsymbol{E}_{c}\right|$, and $\langle\boldsymbol{n} \boldsymbol{n}\rangle \approx \phi \boldsymbol{\delta} / 3$ into Eqs. (22), (34), and (48). This contrasts with the DEM behavior which shows a mild maximum in anisotropy in $\langle\boldsymbol{n} \boldsymbol{n}\rangle_{c}$ in the chain-tilting regime [regime (ii) as defined above]. This discrepancy is presumably related to the failure to capture the phase angle between the $y z$-component of $\langle\boldsymbol{n n}\rangle_{c}$ and the transverse strain in this regime (see Fig. 4). Failure of the DEM to reach complete isotropy in $\langle\boldsymbol{n n}\rangle$ and $\langle\boldsymbol{n n n}\rangle_{c}$ at large $\dot{\gamma}_{\perp}$ is consistent with there being continuing dominance of the contact contribution to the shear stress, discussed above.

We finally present in Fig. 6 plots of the suspension viscosity as functions of $\dot{\gamma}_{r}$ and $\dot{\gamma}_{\perp}$, found by DEM simulation and predicted by the constitutive model (the simulated large $\dot{\gamma}_{r}$ data were previously reported in [22]). The model and DEM simulation both predict that the viscosity reduction obtained under transverse oscillatory shearing is largest for shear-thickened suspensions. This follows naturally from the fact that the oscillations act by breaking up particle-particle contacts: frictional flowing states of $\Pi \gg \Pi^{*}$ are dominated by particle-particle contact stresses and stand to lose a substantially larger proportion of their viscosity by having such contacts removed, compared to lubrication-dominated suspensions $\left(\Pi \ll \Pi^{*}\right)$. For these purposes, the term "shearthickened" suspensions of course includes rate-independent materials of high friction for which $\Pi^{*}$ is effectively zero [3].

Overall, the qualitative agreement between Figs. 6(a) and 6(b) represents encouraging success of our constitutive model under conditions of both rate- and time-dependent flow. Nonetheless, some discrepancies are apparent within the $\left(\dot{\gamma}_{r}, \dot{\gamma}_{\perp}\right)$ range shown here. At small $\dot{\gamma}_{r}$, for which contacts are frictionless and the resulting DEM contact stress is subdominant, the viscosity in DEM simulation is roughly independent of $\dot{\gamma}_{\perp}$. Under these conditions, where the hydrodynamic stress is dominant, it is to be expected that the oscillation-mediated loss of contacts does not lead to a significant change in the viscosity. The constitutive model, meanwhile, predicts a decrease in viscosity with $\dot{\gamma}_{\perp}$ at small $\dot{\gamma}_{r}$. This reflects that changes in the coarse-grained microstructure and the lubrication stress are more pronounced in the constitutive model than in the DEM. At large $\dot{\gamma}_{\perp}$, the viscosity in DEM increases with $\dot{\gamma}_{r}$ since (at this proximity to $\phi_{2}^{J}$ ) the onset of friction leads to a substantial contact stress (albeit lower than when $\dot{\gamma}_{\perp}$ is small). In the constitutive model, however, the viscosity at large $\dot{\gamma}_{\perp}$ is independent of $\dot{\gamma}_{r}$, reflecting that the modeled oscillations over-predict the
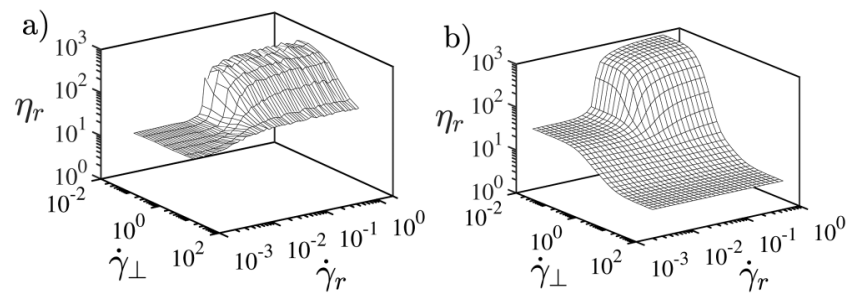

FIG. 6. Suspension viscosity as a function of dimensionless shear rate $\dot{\gamma}_{r}$ and dimensionless frequency $\dot{\gamma}_{\perp}$ for DEM (a) and constitutive model (b). breakup of the microstructure (Fig. 5) and providing further indication that the contact stress is too sensitive to the number of contacts [Eq. (35)].

\section{v. DISCUSSION AND CONCLUSIONS}

We have presented a self-contained derivation for a recently proposed constitutive model for the microstructure and stress of shear-thickening particle suspensions, discussing en route the roles played by the "jamming coordinate" $\xi=\operatorname{Tr}\langle\boldsymbol{n} \boldsymbol{n}\rangle_{c}$ and the contact microstructure $\langle\boldsymbol{n n}\rangle_{c}$, which is related, within the model, to the coarse-grained microstructure tensor $\langle\boldsymbol{n n}\rangle$. This relation allows a closed constitutive model at the coarse-grained level, while making testable predictions for the contact statistics. Along with the stress and other observable quantities, these can be compared with experiment or, as done in this paper, with simulations of particle-based models based on the DEM formalism. The use of DEM simulation data, for which microstructural data can be interrogated almost ad infinitum (in contrast to experiments which generally cannot resolve individual contact forces), offers a set of stringent tests for rheological constitutive models, as emphasized recently by Chacko et al. [20].

In [14], we confronted the new constitutive model with such data for the case of reversal of steady shear flow. The model was found qualitatively correct in most aspects, but with a systematic over-prediction of microstructural anisotropy which was reflected in relatively poor prediction of normal stress differences.

In the present paper, we have taken the simulation-based testing of the model considerably further by addressing steady shear flows with superposed transverse oscillations. For friction-dominated systems $\left(\Pi \gg \Pi^{*}\right)$, this protocol has been shown capable of drastically reducing the mean viscosity, in some cases unjamming systems whose viscosity would otherwise be infinite $[21,22]$. This protocol may find utility in active rheology control [24] for various industrial applications. Because of its strong influence on timedependent suspension microstructure, it provides a range of stringent tests for any constitutive model. Since flow conditions evolve continuously, these tests complement those offered by sudden flow reversal.

Overall, we again found qualitative agreement between the constitutive model predictions and data generated by discrete-element simulation. This applies, in particular, to the decrease in the contacts with increasing oscillation frequency [Fig. 5(b)] and to the transient buildup of the amplitude of the transverse shear stress and its phase relative to the transverse shear rate [Figs. 3(a) and 3(b)].

However, the model falls short in other respects, such as the corresponding phase relation for the $y z$-component of the contact microstructure $\langle\boldsymbol{n n}\rangle_{c}$. This shows a discrepancy that is at least partly the fault of our ansatz for $\langle\boldsymbol{n n}\rangle_{c}$ in terms of $\langle\boldsymbol{n n}\rangle$, given in Eq. (34). Improvement to this ansatz is, therefore, a target for future refinement of our constitutive model. A second discrepancy is that the constitutive model predicts the contact contribution to viscosity to collapse to extremely low levels at high transverse oscillation frequencies so that lubrication terms dominate, whereas the DEM simulations 
show the collapse to be much more moderate, with direct contact terms still dominating the stress, at least when $\phi$ is close to the frictional jamming point $\phi_{2}^{J}$. Third, although Fig. 6 shows broad qualitative agreement for the viscosity as a function of the reduced shear rate $\dot{\gamma}_{r}$ and oscillation frequency $\dot{\gamma}_{\perp}$, the behaviors seen in DEM simulations along both the small $\dot{\gamma}_{r}$ and the large $\dot{\gamma}_{\perp}$ edges of the diagram are not properly captured by the constitutive model.

The explanations of these shortcomings remain a topic of ongoing research, to which we hope to return in future publications. Candidates for improvement include not only the specific approximation for $\langle\boldsymbol{n n}\rangle_{c}$ mentioned above [Eq. (34)], but also the relation between the contact force and the number of contacts $\left[C_{3}\right.$-term in Eq. (5)]; a microstructurebased interaction force with the background $\left[C_{1}\right.$-term in Eq. (5)], which should limit anisotropy in dense systems; the assumption of a friction-independent microstructural evolution [absence of a friction term in Eq. (5)]; the Hinch-Leal type closure relation [Eq. (22)]; and our simplified approach to the angular distribution of birth and death processes among contacts [Eq. (18)].

Pending further exploration of all these aspects, the work reported above already confirms the value of comparing constitutive models for dense suspensions not only with macroscopic experimental observations (which are generally limited to measurements of stress), but also with particlebased simulations that can give detailed microstructural statistics. Such comparisons increasingly allow the assumptions of the model to be tested individually rather than collectively, an approach that we hope should speed future progress toward a fully predictive constitutive rheology for dense suspensions, both shear-thickening and otherwise.

\section{ACKNOWLEDGMENTS}

The authors acknowledge financial support from the Engineering and Physical Sciences Research Council of the United Kingdom (EPSRC) under Grant No. EP/N024915/1, and from the European Research Council under the Horizon 2020 Programme, ERC under Grant agreement No. 740269. M.E.C. is funded by the Royal Society. C.N. is funded by the Maudslay-Butler Research Fellowship at Pembroke College, Cambridge.

\section{REFERENCES}

[1] Mari, R., R. Seto, J. F. Morris, and M. M. Denn, "Shear thickening, frictionless and frictional rheologies in non-Brownian suspensions," J. Rheol. 58, 1693-1724 (2014).

[2] Seto, R., R. Mari, J. F. Morris, and M. M. Denn, "Discontinuous shear thickening of frictional hard-sphere suspensions," Phys. Rev. Lett. 111, 218301 (2013).

[3] Guy, B. M., M. Hermes, and W. C. K. Poon, "Towards a unified description of the rheology of hard-particle suspensions," Phys. Rev. Lett. 115, 088304 (2015).

[4] Lin, N. Y. C., B. M. Guy, M. Hermes, C. Ness, J. Sun, W. C. K. Poon, and I. Cohen, "Hydrodynamic and contact contributions to continuous shear thickening in colloidal suspensions," Phys. Rev. Lett. 115, 228304 (2015).
[5] Royer, J. R., D. L. Blair, and S. D. Hudson, "Rheological signature of frictional interactions in shear thickening suspensions," Phys. Rev. Lett. 116, 188301 (2016).

[6] Wagner, N. J., and J. F. Brady, "Shear thickening in colloidal dispersions," Phys. Today 62(10), 27-32 (2009).

[7] Jamali, S., and J. F. Brady, "Alternative frictional model for discontinuous shear thickening of dense suspensions: Hydrodynamics," Phys. Rev. Lett. 123, 138002 (2019).

[8] Wyart, M., and M. E. Cates, "Discontinuous shear thickening without inertia in dense non-Brownian suspensions," Phys. Rev. Lett. 112, 098302 (2014).

[9] Boyer, F., É. Guazzelli, and O. Pouliquen, "Unifying suspension and granular rheology," Phys. Rev. Lett. 107, 188301 (2011).

[10] Comtet, J., G. Chatté, A. Niguès, L. Bocquet, A. Siria, and A. Colin, "Pairwise frictional profile between particles determines discontinuous shear thickening transition in non-colloidal suspensions," Nat. Commun. 8, 15633 (2017).

[11] Krieger, I. M., and T. J. Dougherty, "A mechanism for non-Newtonian flow in suspensions of rigid spheres," Trans. Soc. Rheol. 3, 137-152 (1959).

[12] Hermes, M., B. M. Guy, W. C. K. Poon, G. Poy, M. E. Cates, and M. Wyart, "Unsteady flow and particle migration in dense, non-Brownian suspensions," J. Rheol. 60, 905-916 (2016).

[13] Guy, B. M., C. Ness, M. Hermes, L. J. Sawiak, J. Sun, and W. C. K. Poon, "Testing the Wyart-Cates model for non-Brownian shear thickening using bidisperse suspensions," Soft. Matter 16, 229-237 (2020).

[14] Gillissen, J. J. J., C. Ness, J. D. Peterson, H. J. Wilson, and M. E. Cates, "Constitutive model for time-dependent flows of shearthickening suspensions," Phys. Rev. Lett. 123, 214504 (2019).

[15] Gillissen, J. J. J., and H. J. Wilson, "Modeling sphere suspension microstructure and stress," Phys. Rev. E 98, 033119 (2018).

[16] Gadala-Maria, F., and A. Acrivos, "Shear-induced structure in a concentrated suspension of solid spheres," J. Rheol. 24, 799-814 (1980).

[17] Ness, C., and J. Sun, "Two-scale evolution during shear reversal in dense suspensions," Phys. Rev. E 93, 012604 (2016).

[18] Peters, F., G. Giovanni, S. Gallier, F. Blanc, E. Lemaire, and L. Lobry, "Rheology of non-Brownian suspensions of rough frictional particles under shear reversal: A numerical study," J. Rheol. 60, 715-732 (2016).

[19] Goddard, J. D., "A dissipative anisotropic fluid model for non-colloidal particle dispersions," J. Fluid Mech. 568, 1-17 (2006).

[20] Chacko, R. N., R. Mari, S. M. Fielding, and M. E. Cates, "Shear reversal in dense suspensions: The challenge to fabric evolution models from simulation data," J. Fluid Mech. 847, 700-734 (2018).

[21] Lin, N. Y. C., C. Ness, M. E. Cates, J. Sun, and I. Cohen, "Tunable shear thickening in suspensions," Proc. Natl. Acad. Sci. U.S.A. 113, 10774-10778 (2016).

[22] Ness, C., R. Mari, and M. E. Cates, "Shaken and stirred: Random organization reduces viscosity and dissipation in granular suspensions," Sci. Adv. 4, eaar3296 (2018).

[23] Cates, M. E., J. P. Wittmer, J.-P. Bouchaud, and Ph. Claudin, "Jamming, force chains, and fragile matter," Phys. Rev. Lett. 81, 1841 (1998).

[24] Sehgal, P., M. Ramaswamy, I. Cohen, and B. J. Kirby, "Using acoustic perturbations to dynamically tune shear thickening in colloidal suspensions," Phys. Rev. Lett. 123, 128001 (2019).

[25] Gillissen, J. J. J., and H. J. Wilson, "Effect of normal contact forces on the stress in shear rate invariant particle suspensions," Phys. Rev. Fluids 4, 013301 (2019).

[26] Kim, S., and S. Karrila, Microhydrodynamics: Principles and Selected Applications (Butterworth-Heinemann, Boston, 1991). 
[27] Seto, R., and G. G. Giusteri, "Normal stress differences in dense suspensions," J. Fluid Mech. 857, 200-215 (2018).

[28] Hinch, E. J., and L. G. Leal, "Constitutive equations in suspension mechanics. Part 2. Approximate forms for a suspension of rigid particles affected by Brownian rotations," J. Fluid Mech. 76, 187-208 (1976).

[29] Jeffrey, D. J., and Y. Onishi, "Calculation of the resistance and mobility functions for two unequal rigid spheres in low-Reynolds-number flow," J. Fluid Mech. 139, 261-290 (1984).

[30] Jeffrey, D. J., "The calculation of the low Reynolds number resistance functions for two unequal spheres," Phys. Fluids 4, 16-29 (1992).
[31] Ball, R. C., and J. R. Melrose, "A simulation technique for many spheres in quasi-static motion under frame-invariant pair drag and Brownian forces," Physica A 247, 444-472 (1997).

[32] Cheal, O., and C. Ness, "Rheology of dense granular suspensions under extensional flow," J. Rheol. 62, 501-512 (2018).

[33] Cundall, P. A., and O. D. L. Strack, "A discrete numerical model for granular assemblies," Geotechnique 29, 47-65 (1979).

[34] Plimpton, S., "Fast parallel algorithms for short-range molecular dynamics," J. Comput. Phys. 117, 1-19 (1995). 